\section{Weapons research}

\section{Sandia's nuclear simulation}

\section{Albuquerque, New Mexico}

A NEW $\$ 39$ million laboratory that will significantly increase the ability of nuclear weapons designers to simulate the effect of radiation on critical nuclear components is under construction at Sandia National Laboratories. The centrepiece of the new laboratory is a 20 trillion $\left(20 \times 10^{12}\right)$ watt gamma-ray source to simulate the intense short pulse of radiation from a nuclear explosion that can disable the electronics necessary for arming and fusing nuclear weapons as they approach their targets. A 16.5 trillion watt $X$-ray source will also be available at the new Simulation Testing Laboratory through the recycling of Sandia's Pulsed Beam Fusion Accelerator (PBFA-I), at present used for inertial confinement fusion research. The laboratory will be completed in 1987.

Sandia, which has responsibility for the engineering of nuclear weapons, has been heavily involved in the development of "hardened" electronic components. This work has been prompted by concern that the credibility of the United States deterrent would be lost if its nuclear weapons were vulnerable to countermeasures such as the preemptive detonation of a nuclear bomb. The technical challenge in simulation testing has been to create in the laboratory a pulse of gamma and $X$ radiation that is both intense and short. Conventional pulsed power technology faces an intrinsic obstacle: methods used to increase current also tend to increase inductance and pulse length.

Sandia's solution, which will permit its new gamma-ray machine, Hermes-III, to produce a pulse of 1 million amperes at 15 million volts in a mere 20 nanoseconds, involves a technology that has implications for controlled fusion, nuclear weapons design and also particle-beam weapons all of which are intimately wrapped together in the laboratory's pulsed power research (see below). The technique is to split the pulse generator into small nodules, which are combined in series or parallel to yield either high voltages or high currents while keeping inductance small.

The result is that Hermes-III will produce 10 times the power of the best single-module machine (Hermes-II, built in 1967, and still Sandia's work-horse for gamma-ray simulation) in a pulse less than one-tenth as long. PBFA-I, when adapted for X-ray simulation, will offer a similar improvement over the laboratory's present $\mathrm{X}$-ray simulator.

The multiple-module concept was first applied in the Department of Defense's "Aurora" machine, which runs four pulse generators in parallel to produce a high current. Sandia then built a series of machines improving on this multiplemodule principle. As well as their use in $\mathrm{X}$-ray simulation, they also paved the way for particle-beam fusion research, which PBFA-I was designed to explore. PBFA-I uses 36 parallel generators arranged like spokes of a wheel, converging on a target at the hub. It generates 15 million amps at 2 million volts.

Although the combination of high current and short pulse offered by the parallel module arrangement is equally applicable to gamma-ray production, high voltage is needed also to generate the more energetic gamma rays. Gerold Yonas, director of Sandia's pulsed power research, says that in 1975 a Soviet publication suggested the possibility of tying modules together in series to produce such high voltages (of the order of $10 \mathrm{MV}$ are needed). Sandia conducted a joint project with the Air Force Weapons Laboratory in 1978 to prove the feasibility of the concept, which in effect involves creating a linear accelerator out of the modules. That is the technology that will be utilized in HermesIII and which is now being further developed in a test-bed called MABE.

Sandia scientists are quick to insist that

in spite of these improvements, laboratory simulation will never be a complete substitute for testing with actual nuclear explosions, as now carried out at the Nevada test site. Yonas says that the extrapolations of the laboratory tests to the actual spectral distribution of radiation produced in a nuclear blast and to the actual pulse and decay rates are necessarily problematical. But the actual insistence on "proof-testing" under the more realistic conditions at Nevada may have more to do with nuclear strategy than scientific certainty. "Deterrence is nothing but peception", Yonas says. "Ultimately, the credibility of our deterrence lies in a proof test."

Laboratory simulation does permit a more controlled approach to understanding radiation effects on electronic components; it is also cheaper than setting off nuclear explosions and can be done much more often. Sandia's new facilities will be used chiefly to test the radiation hardness of nuclear weapon electronics; the laboratory also expects to continue a modest amount of contract work for the Department of Defense, testing electronic systems in battlefield equipment.

Stephen Budiansky

\title{
Mixed mission brings stability
}

SANDIA National Laboratories, founded in 1945 to provide engineering support for the Manhattan Project at Los Alamos, continues to be chiefly concerned with weapons research, development and testing. Its increasing strength in basic science, however, particularly in the pulsed power research programme, has given the laboratory something of a split personality.

The entanglement of interests is perhaps most apparent in the inertial confinement fusion (ICF) programme at Sandia. Begun in the 1970s, the ICF programme was at first justified chiefly by the promise of commercial fusion energy. The programme was, however, always supported by the office of military applications at the Department of Energy and its predecessor agencies. And now that ICF has lost its bloom, with magnetic confinement fusion emerging as the more practical route to commercial energy production, its status is even more confused.

A new ICF machine, PBPA-II, which will combine the parallel and series arrangement of modules to produce $\mathbf{1 0 0}$ trillion watts at as much as $24 \mathrm{MV}$ in a puise 13 nanoseconds long, is in many ways designed with controlled fusion research in mind. The decision to go to higher voltage was prompted by the choice of lithium ions rather than protons for the particle beam, which is focused on a deuterium-tritium pellet, lithium ions can be more easily focused, giving greater power density.

On the other hand, programme-director Yonas concedes that "our choices have been influenced primarily by our short- term goals," which include studies of pellet shapes with significance for nuclear weapons design. PBFA-II will, however, be unclassified, except when a "classified pellet"' is being tested.

The simulation programme has similarly been intimately wrapped up with the ICF research and the same may happen to socalled "star wars", or particle-beam weapons. Although Yonas says that PBFA-II "would be a loser" for particle beam weapons research, the basic principles of pulsed power are clearly applicable.

Sandia's combination of a primary weapons mission with a broad expertise in energy sciences and microelectronics has been a formula for financial stability. When the Reagan Administration cut energy research by 40 per cent, the laboratory's executive vice-president Albert Narath says, Sandia had little trouble shifting affected staff members to defence research.

The laboratory's current budget of $\$ 874$ million (with 8,087 full-time employees) has remained fairly level in recent years, with 60 per cent devoted to nuclear weapons activities. Narath thinks there may be a major shift if the "star wars" initiative materializes: "We are thinking very hard about the possibility of transitioning to a defence-dominated deterrence." Sandia's expertise in pulsed power and in hardened microelectronics would place the laboratory in a "very good" position for contributing to a star wars initiative, says Narath.
Stephen Budiansky 